\title{
Factores predisponentes en relajación residual neuromuscular
}

\author{
María Dolores Torres Soto ${ }^{1}$, Aurora Torres Soto ${ }^{2}$, María de la Luz Torres Soto ${ }^{3}$, \\ Leticia Bermudez Rosales ${ }^{3}$, Eunice E. Ponce de León Sentí ${ }^{2}$ \\ ${ }^{1}$ Universidad Autónoma de Aguascalientes, Departamento de Sistemas de Información, \\ Aguascalientes, México \\ ${ }^{2}$ Universidad Autónoma de Aguascalientes, Departamento de Ciencias de la Computación, \\ Aguascalientes, México \\ ${ }^{3}$ Centenario Hospital Miguel Hidalgo, \\ Aguascalientes, México \\ mdtorres@correo.uaa.mx, \{atorres, eponce\}@correo.uaa.mx, \\ \{tishats, letybermudez3\}@hotmail.com
}

Resumen. Este artículo muestra los resultados del seguimiento y análisis de 210 pacientes que ingresaron a la Unidad de Cuidados Post-anestésicos del Centenario Hospital Miguel Hidalgo de Abril a Septiembre de 2014 en la ciudad de Aguascalientes. La herramienta empleada para la identificación de los factores predisponentes de relajación residual neuromuscular fue un algoritmo genético hibridizado con los conceptos de testor y testor típico, así como la integración de operadores genéticos que permiten la generación y mejora de individuos prometedores durante el proceso. La relajación residual neuromuscular es la condición clínica determinada por la persistencia de los efectos farmacológicos de los bloqueantes neuromusculares; situación que debe ser evitada debido a que condiciona el aumento de la morbimortalidad del paciente. Los resultados sugieren como factores predisponentes de relajación muscular residual, variables como el género y la duración de los procedimientos (que no han sido reportadas en la literatura), entre otras.

Palabras clave: algoritmo genético, testores típicos, relajación residual neuromuscular, factores predisponentes.

\section{Introducción}

La relajación residual neuromuscular (RRNM) es una condición clínica, determinada por la persistencia de los efectos farmacológicos de los bloqueantes neuromusculares; que puede aumentar la morbilidad postoperatoria con incidencia variable entre $0 \%$ y $93 \%$ [16]. El propósito de este estudio, es la identificación de sus factores predisponentes mediante el uso de una herramienta genética híbrida. 
La determinación del conjunto de características que mejor describe un fenómeno, es un problema bien conocido y abordado mediante diferentes tipos de herramientas. Algunas extraídas de la minería de datos [15] y otras, debido a su conocido nivel de complejidad, inspiradas en técnicas no exhaustivas como es el caso de metaheurísticas tanto mono-objetivo [19, 22, 23, 24], como multiobjetivo [13]. La determinación de un subconjunto de características tiene un espacio de búsqueda de $2^{\mathrm{n}}$ posibles subconjuntos, por lo que se ha catalogado como un problema NP-duro. Para una revisión detallada sobre los marcos de trabajo para éste problema, el lector puede remitirse a [6].

Es importante hacer mención de que para nuestra aplicación, una característica equivale a una variable descriptora de la condición médica del paciente, por tal motivo, en el resto del documento haremos uso indistinto de los términos variable y característica.

El algoritmo que se empleó en este trabajo, está definido dentro de un marco de trabajo adaptable tanto para aprendizaje supervisado como no supervisado [23], además de la inclusión de los operadores de mejora y de aceleración. El algoritmo es conducido mediante la valoración desde una perspectiva de testor de los individuos generados, consiguiendo una reducción considerable del fenómeno en descripción.

El algoritmo presentado en este documento se basa en el conocido como "algoritmo genético simple" de Goldberg [8], cuyas bases aparecieron a mediados de los años setentas entre Holland y sus colaboradores [9]. La idea central de esta metaheurística está inspirada en la supervivencia de los individuos más aptos, de acuerdo a la teoría de la evolución.

Este trabajo se ha organizado de la siguiente manera: en la sección 2 se discuten algunos conceptos básicos relacionados tanto con la técnica empleada para la solución del problema, como con el problema de relajación residual neuromuscular. En la sección 3, se presenta la descripción del algoritmo empleado para la identificación de los factores predisponentes de RRNM, así como la explicación del operador de aceleración. Los resultados obtenidos son descritos y discutidos en la sección 4, para finalmente mostrar las conclusiones y trabajo futuro en la sección 5.

\section{Conceptos básicos}

\subsection{Testores y testores típicos}

El concepto de testor aparece a mediados de los años cincuenta (1950's) [3]. Al principio, los testores típicos se usaron como una herramienta muy eficaz para detectar fallas en circuitos eléctricos. Posteriormente, se usaron para llevar a cabo clasificación supervisada y selección de variables en problemas de geología [1]. El uso que se les da en este trabajo a los testores y testores típicos está estrechamente relacionado con selección de características, cuyo precursor es Dmitriev, Zhuravlev y colaboradores [5].

En esencia, un testor es un subconjunto de características que distinguen objetos provenientes de clases distintas. De acuerdo con Santiesteban y Pons en 2003 [18], así como Shulcloper en 1995 [20], un testor típico es un testor al que ya no se le puede 
quitar ninguna de sus características sin que pierda su condición de testor. Dicho de otra manera, un testor es todavía redundante en términos de que aún puede ser un conjunto más pequeño de características pudiendo identificar la clase a la que pertenece el objeto en cuestión; mientras que un testor típico está ya formado por el conjunto de características mínimo necesario para garantizar aún la identificación de la clase a la que pertenece un objeto específico.

Supongamos que $\mathbf{U}$ es una colección de objetos, y esos objetos son descritos por un conjunto $\mathbf{n}$ de características, los objetos, se encuentran agrupados en $\mathbf{k}$ clases. Comparando cada par de características que pertenecen a objetos en clases diferentes haciendo uso de algún criterio de comparación, podemos obtener la Matriz de diferencias (MD). Esta matriz de diferencias puede ser muy grande cuando son encontradas muchas diferencias entre los miembros de una clase contra los de las demás.

Llamémosle $\mathrm{T}$ a un subconjunto del total de etiquetas de columna de MD. Llamaremos Matriz Básica (MB) a un conjunto de datos especial obtenido de la eliminación de todas las filas pertenecientes a la MD que no son básicas.

Supóngase que a y b son dos filas de la matriz de diferencias, a es una subfila de $\mathbf{b}$ si $\left(\forall_{\mathbf{i}} \mid \mathbf{b}_{\mathbf{i}}=\mathbf{0}, \mathbf{a}_{\mathbf{i}}=\mathbf{0}\right)$ y además las filas cumplen al menos en una de sus columnas (i $\mid$ $\left.\mathbf{b}_{\mathbf{i}}=\mathbf{1} \Lambda \mathbf{a}_{\mathbf{i}}=\mathbf{0}\right)$ [11]. En otras palabras, a es una fila básica de MD si no hay ninguna otra fila menor que a en MD.

Dada una Matriz de diferencias MD, llamaremos Matriz Básica (MB) a la matriz formada exclusivamente por filas básicas de MD [11].

T es un testor de la MA (Matriz de aprendizaje) si no existen filas de ceros en la MB al eliminar de ésta todas las columnas que no pertenezcan al conjunto $\mathbf{T}$.

El conjunto $\mathbf{T}$ es típico si al eliminar cualquiera de sus características $\mathbf{j} \mid \mathbf{j} \in \mathbf{T}, \mathbf{T}$ deja de ser testor.

El conjunto de todos los testores típicos de la MD es igual al conjunto de todos los testores típicos de la MB [11].

Calcular todos los testores típicos es un procedimiento muy costoso; de hecho, todos los algoritmos exhaustivos conocidos tienen una complejidad exponencial e incluso dependen del tamaño de la matriz [17].

El lector interesado podrá revisar algunos de los algoritmos desarrollados para el cálculo del conjunto completo de testores típicos como: BT [21], TB [21], CT-EXT [17], LEX [18] y REC [20] entre otros.

En este trabajo, utilizamos un algoritmo genético que usa testores típicos para encontrar las variables más importantes en relajación residual, así como su peso relativo en términos de su capacidad predictiva. Algunos conceptos e ideas relacionados con la teoría de testores típicos puede ser consultada por el lector en [12].

\subsection{Relajación residual neuromuscular}

La necesidad de incorporar nuevas guías o estrategias en la buena práctica de uso de los bloqueantes neuromusculares es una necesidad imperiosa en Anestesiología. En la práctica actual sólo existen recomendaciones de actuación con el propósito de convencer de que la monitorización neuromuscular es una herramienta muy útil para el buen uso de los bloqueadores neuromusculares. El único método satisfactorio para 
determinar un grado de bloqueo neuromuscular es estimular un nervio motor con una corriente eléctrica y observar el grado de contracción de los músculos inervados por ese nervio. Es fundamental encontrar alternativas que permitan prever problemas en pacientes con predisposición a la relajación residual neuromuscular.

La razón por la que la monitorización neuromuscular no ha recibido la aceptación necesaria en la práctica clínica es un reflejo de la discordancia entre lo que la literatura recomienda y lo que los clínicos pueden medir. En la actualidad, la monitorización de la función neuromuscular, no es una práctica común porque los clínicos no saben interpretar correctamente sus resultados [2]. El uso de un neuroestimulador es más una excepción que una regla en cualquier servicio de anestesia en nuestro país [2]. Podemos afirmar que la relajación residual neuromuscular (RRNM) es una lección que no ha sido asimilada en su importancia en las instituciones de salud mexicanas, es un tópico al que se le menosprecia y al que no se ha dado el valor que merece [14].

A pesar de la alta incidencia de relajación residual neuromuscular (RRNM) en la unidad de cuidados postanestésicos (UCPA), en algunos estudios se reporta de hasta $24 \%$ [14], el uso del monitoreo de la función neuromuscular ya sea en cirugía o en recuperación es infrecuente en demasía.

\subsection{Monitorización / estimulación}

La posible toxicidad de los bloqueadores neuromusculares es alta ya que interfieren con la función del sistema respiratorio.

La depresión ventilatoria es una causa importante de mortalidad y morbilidad relacionadas con la anestesia [14], y no hay duda de que el bloqueo neuromuscular residual en estas circunstancias es un factor importante. Además, los límites de seguridad son reducidos pues el bloqueo se produce en un margen estrecho de ocupación de receptores. Es por eso que es importante que el clínico valore el efecto de los bloqueadores neuromusculares sin la influencia distractora de los agentes volátiles, anestésicos intravenosos y opioides. [7]

El monitoreo de la función neuromuscular tiene las siguientes utilidades:

a) Administración de la dosis óptima individual de relajantes muscular y antídotos

b) Administración de estos medicamentos en el momento adecuado e identificar el momento de reversión

c) Identificación del tipo de relajante en caso de bloqueo residual. Por tanto, se evita la sobredosis, se previene el riesgo de subdosificación - que puede ser de graves consecuencias en neurocirugía o en cirugía de cámara anterior del globo ocular abierta, entre otras, permite administrar el anticolinesterásico en el momento adecuado, se utiliza menor cantidad de medicamento, se evita el riesgo de relajación prolongada, y en consecuencia la recuperación es más rápida. Una alternativa importante es conocer con antelación las características del paciente que le predisponen fisiológicamente a manifestar una relajación residual neuromuscular para tener precauciones especiales con esos casos aun cuando no se cuente con el neuroestimulador (que es un equipo de monitorización costoso). 


\subsection{Selección de características}

Selección de Subconjuntos de Características (FSS por sus siglas en inglés) es un tópico frecuentemente usado para reducir dimensionalidad. En este sentido existen dos grupos de autores que pueden ser ampliamente identificados: aquellos quienes consideran la importancia o relevancia de las variables, así como la no redundancia de las mismas [4] y aquellos que consideran la importancia de distinguir entre las características óptimas y las relevantes [10]. Los dos métodos más populares para realizar selección de características son los métodos Wrapper y Filter [15], [10].

\section{Algoritmo utilizado}

\subsection{Marco de trabajo}

El marco de trabajo de la presente investigación se muestra en el diagrama de la Fig. 1.

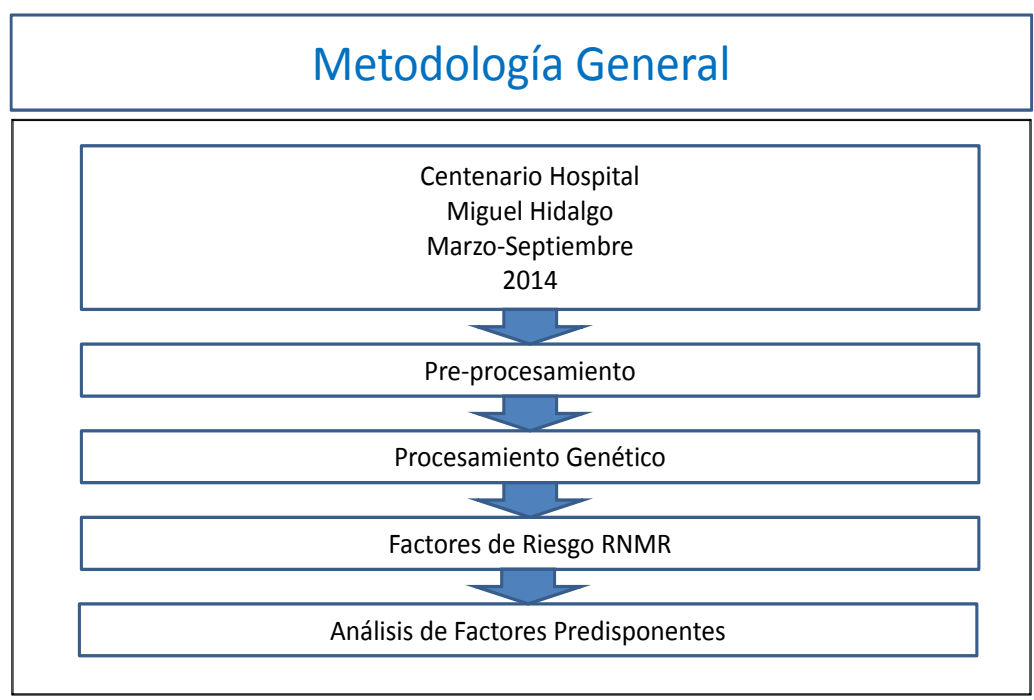

Fig. 1. Marco de trabajo

Como se puede ver en la Fig. 1, se colectó la información de todos los casos que fueron autorizados para ser monitorizados en el turno matutino de Marzo a Septiembre de 2014 en el Centenario Hospital Miguel Hidalgo de la ciudad de Aguascalientes, México. Con esta información, se realizó un pre-procesamiento que permitió reducir el conjunto de variables de 25 a 16. Posteriormente, se realizó el procesamiento genético descrito en la sección 3.3 y se redujo el conjunto a 10 variables que fueron analizadas de manera más profunda. Y finalmente, el personal médico realiza una valoración y análisis de los resultados del algoritmo ejecutado. 


\subsection{Operador de aceleración y mecanismo de mejora}

Es un operador de aceleración que permite que los individuos que se van generando de manera aleatoria, se manipulen para que se mantengan en el espacio de soluciones de testores, ahorrando recursos computacionales.

Este operador fue desarrollado por Torres (2010) [23] y permite hacer un análisis simple de la matriz básica para dirigir la creación de individuos hacia un espacio de soluciones con cierta calidad garantizada.

El mecanismo de mejora se presenta en (Torres, 2010) [23] y consiste en una mutación inteligente, que agrega o elimina bits de para encontrar el testor típico dentro de un testor (que no es típico) o para encontrar el testor partiendo de una cadena que no califica como testor.

\subsection{Pseudocódigo}

El siguiente pseudocódigo representa el comportamiento general del algoritmo Evolutivo Híbrido realizado para la identificación de las principales variables que predisponen a la presentación de relajación residual neuromuscular.

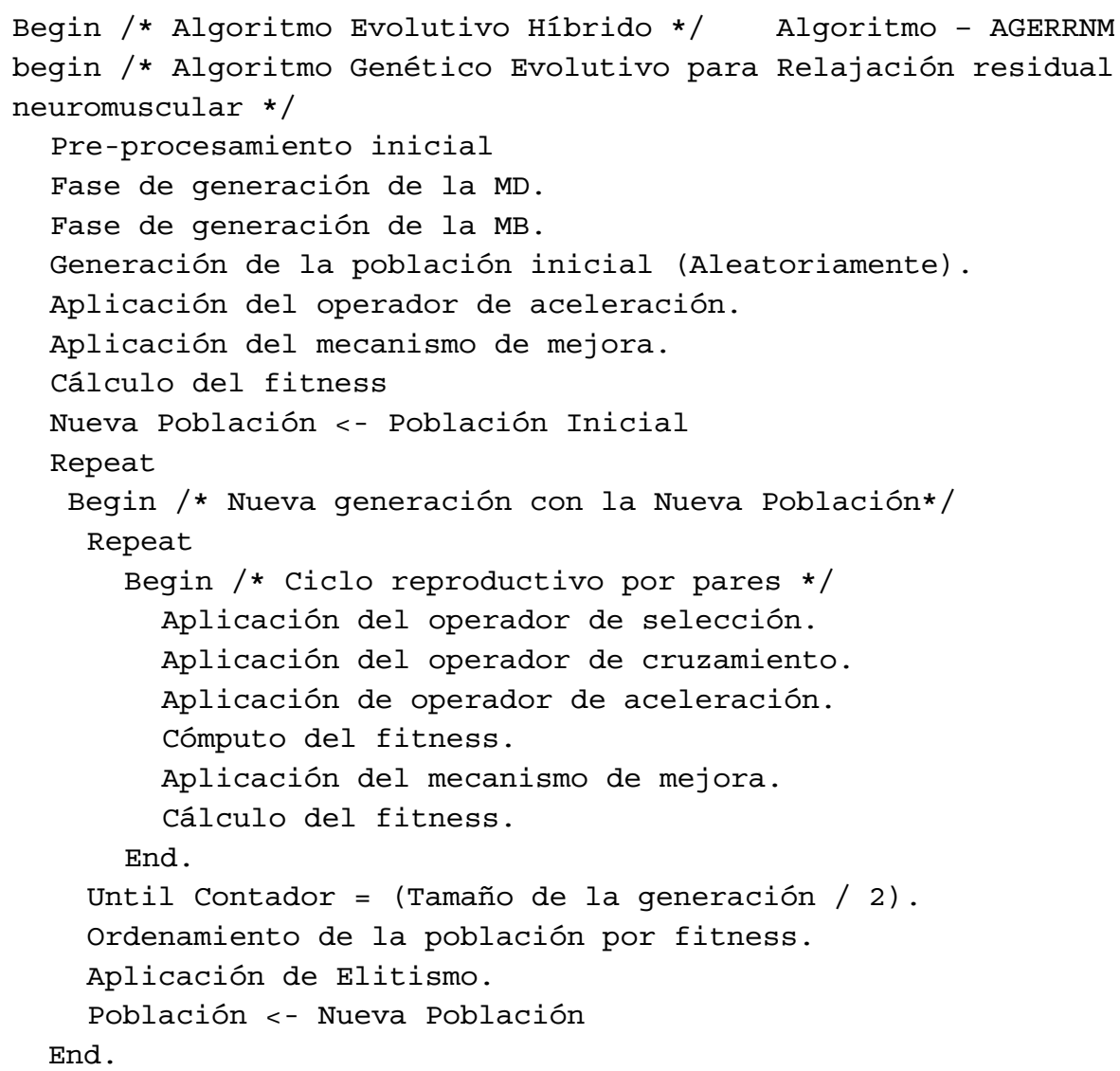




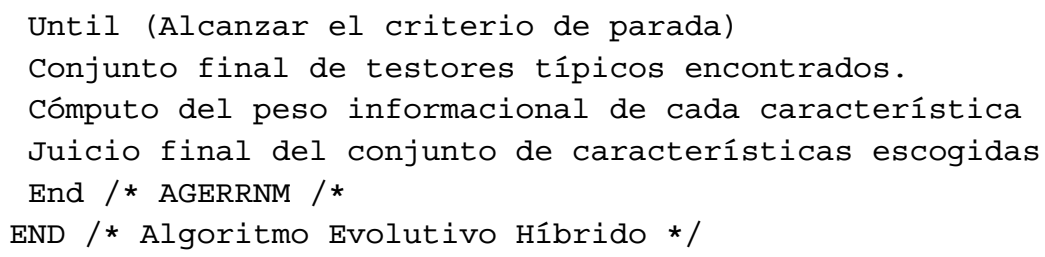

El algoritmo mostrado es un algoritmo genético (AG) en el que se integraron 500 individuos por generación y que fue ejecutado por 300 generaciones usando una probabilidad de cruzamiento alta $(80 \%)$, cuya mutación es inteligente (mecanismo de mejora) es aplicada en el $100 \%$ de los individuos y el operador de aceleración también.

\section{Experimentos y resultados}

Se evaluaron un total de 210 pacientes que ingresaron a la Unidad de cuidados postanestésicos (UCPA) del Centenario Hospital Miguel Hidalgo (CHMH) que cumplieron con los criterios de selección. 46 pacientes tuvieron relajación residual neuromuscular (RRNM) a su ingreso a la UCPA (21.9\%).

Como se mencionó anteriormente, la RRNM es un efecto indeseable en pacientes que salen de un procedimiento quirúrgico porque pueden caer en amnea (insuficiencia respiratoria), si se marean y vomitan, pueden brinco-aspirar, etc.

Esta condición aumenta en general el índice de morbimortalidad pues el paciente no se encuentra completamente recuperados de los relajantes musculares usados en anestesia.

Tabla 1. Peso informacional de las variables arrojadas por el algoritmo

\begin{tabular}{|c|c|c|}
\hline No. & Variable & $\begin{array}{c}\text { Peso Informacio- } \\
\text { nal }\end{array}$ \\
\hline 1 & Género & $100 \%$ \\
\hline 2 & Anestésico & $48 \%$ \\
\hline 3 & Oximetría & $40 \%$ \\
\hline 4 & Clasificación peso & $39 \%$ \\
\hline 5 & Temperatura & $39 \%$ \\
\hline 6 & Tiempo Anestésico & $37 \%$ \\
\hline 7 & Relajante & $36 \%$ \\
\hline 8 & Monitoreo & $36 \%$ \\
\hline 9 & Clasificación por edad & $29 \%$ \\
\hline 10 & Reversión & $29 \%$ \\
\hline
\end{tabular}

Después de ejecutar el algoritmo mostrado en la sección 3.3, pudimos reducir las variables que predisponen la relajación residual neuromuscular de 26 hasta 10 al final 
del proceso. La calificación en términos del peso informacional, (o sea el nivel de importancia de las variables para predecir la relajación residual neuromuscular) se muestra en la Tabla 1

Aunque la cantidad de mujeres intervenidas quirúrgicamente en el Centenario Hospital Miguel Hidalgo es mayor que la de hombres, tuvimos un peso informacional de $100 \%$ en la variable género como resultado del algoritmo evolutivo basado en testores.

Este resultado generó sorpresa en el personal médico de apoyo a la investigación, sin embargo, al revisar la matriz de entrenamiento de intervenciones quirúrgicas, pudo observarse que en efecto, el porcentaje de las mujeres que tuvieron RRNM (relajación neuromuscular) de $24.82 \%$ (35 de 141), es significativamente mayor que el de los hombres $18.64 \%$ (11 de 69). Por lo tanto, podemos aceptar que el hecho de ser mujer efectivamente aumenta el riesgo de presentar RRNM. Los médicos pudieron encontrar una explicación práctica a este fenómeno: el tejido adiposo de las mujeres es mayor al de los hombres y los relajantes se absorben en la grasa y se liberan lentamente de este tipo de tejido.

El algoritmo evolutivo arrojó un peso informacional del $29 \%$ para la variable edad. Por lo tanto se realizó un análisis de estos resultados y se pudo observar que de los siete pacientes de 12 o menos años analizados en nuestra muestra, ninguno presentó RRNM, esto puede interpretarse como que el riesgo de RRNM aumentará con la edad y disminuye nuevamente en edades avanzadas.

El peso informacional que arrojó la variable ASA (Referente al estado general de salud del paciente) fue de $28 \%$. Al analizar más detenidamente esta variable, observamos que los porcentajes de RRNM fueron $24.39 \%$ (20 de 82) para los pacientes ASA $1,18.69 \%$ (20 de 107) para ASA 11 y $28.57 \%$ (6 de 21 ) para ASA III. Este resultado nos inclinaría a asumir que el hecho de tener clasificaciones de ASA III, eleva el riesgo de presentar RRNM.

El índice de masa corporal se clasificó de acuerdo al peso corporal en 4 categorías: Bajo peso, normal, sobrepeso y obesidad.

El peso informacional de la variable IMC (Índice de Masa Corporal) que integra peso y talla del paciente, resultó en $39 \%$ por lo que analizamos los resultados de la siguiente manera: El porcentaje de RRNM en pacientes de peso bajo es de $37.5 \%$ (3 de 8 ); el de pacientes con peso normal es de $28.75 \%$ ( 23 de 80 ); para los pacientes con sobrepeso el porcentaje es de $14.66 \%$ (11 de 75$)$; por último para los pacientes con obesidad el porcentaje es de 19.1\% (9 de 47). Asumimos en base a este resultado, que el riesgo de presentar RRNM tiene una relación inversa con el peso del paciente, probablemente porque a mayor peso corporal, la dosis de relajantes musculares se calcula de acuerdo al peso ideal, de tal forma que los pacientes con peso bajo o normal reciben mayor cantidad en proporción a su peso de relajantes.

En términos de la variable temperatura, el personal médico corroboró los resultados del algoritmo, pues está reportado en la literatura que la hipotermia potencializa el efecto de los relajantes musculares.

El peso informacional de la variable tipo de anestésico utilizado arrojado por el algoritmo fue de $48 \%$. Este peso informacional es alto, aunque el personal médico atribuyó su peso al hecho de que de los 2 pacientes que se manejaron con propofol y 
fentanil, uno presentó RRNM (50\%), y el único paciente que se manejó con una combinación de gases anestésicos presentó el evento(100\%). En vista de esto, los médicos fueron muy reservados para aseverar que la combinación de gases anestésicos o la técnica de Anestesia endovenosa total son determinantes de riesgo para presentar RRNM.

La variable administración de anestésicos locales transoperatoria tuvo un peso informacional del $28 \%$. El porcentaje de pacientes a los que se les aplicaron anestésicos locales en algún momento de la cirugía y que tuvieron RRNM es de 20.83\% (15 de 72) y el de los pacientes que no los recibieron es de $22.46 \%$ (31 de 138). A la luz de estos resultados no podemos considerar que la administración de anestésicos locales durante el procedimiento anestésico sea un factor de riesgo para RRNM en esta investigación.

Otra variable de gran interés fue el tipo de relajante muscular utilizado, se puede caer en el error de pensar que el cisatracurio fue el relajante de mayor riesgo para condicionar RRNM, ya que es el más utilizado en el Centenario Hospital Miguel Hidalgo. Al analizar el porcentaje de pacientes manejados con cisatracurio y que tuvieron RRNM es de $18.88 \%$ (34 de 180). Pero al analizar el porcentaje de los pacientes manejados con vecuronio observamos que se dispara al 36\%(9 de 25). El único paciente manejado con rocuronio tuvo RRNM 100\%; y de los tres pacientes manejados con la combinación de succinilcolina y un relajante no despolarizante, el $66.66 \%$ (2 de 3) presentaron RRNM. Por último, el único paciente manejado con una combinación de dos relajantes no despolarizantes, no tuvo RRNM. De lo anterior, podemos asumir que el riesgo de tener RRNM se relaciona en mayor medida al uso de relajantes musculares derivados de esteroides y a la combinación de succinilcolina con un despolarizante, pero este fenómeno deberá estudiarse de manera dirigida.

El monitoreo de la relajación muscular transoperatoria protege a los pacientes de sufrir RRNM presentando un peso informacional del 36\%.

La reversión farmacológica transoperatoria del efecto del relajante neuromuscular, presentó un peso informacional de $29 \%$. El porcentaje de pacientes que fueron revertidos y que presentaron RRNM es de 32\% (8 de 17) contra el de los no revertidos y que también presentaron RRNM 20.5\% (38 de 185). Estos resultados son alarmantes, porque la finalidad de revertir farmacológicamente el efecto de los relajantes musculares es precisamente para evitar la RRNM, lo que no se está logrando.

De lo anterior, el hecho de revertir farmacológicamente el efecto del relajante neuromuscular durante el transanestésico, no previene el evento de RRNM a menos que se monitorice objetivamente la relajación.

El peso informacional de la oximetría calculado por el algoritmo genético fue de $40 \%$. El porcentaje de los pacientes que tuvieron registros de oximetría de pulso de $90 \%$ y más (no hipoxemia) y que presentaron RRNM fue de $21.27 \%$ (40 de 188). El porcentaje de los pacientes con evidencia de hipoxemia (menor de 90\%) y con RRNM fue de $27.2 \%$ ( 6 de 22$)$.

El peso informacional de la variable tiempos anestésicos, fue de $37 \%$ y su análisis es el siguiente: el porcentaje de los pacientes con tiempos anestésicos de una hora o menos y que presentaron RRNM fue de 35\% (7 de 20); el de los pacientes con tiempos anestésicos de 1 a dos horas fue de 24.73\% (23 de 93); por último el de los pa- 
cientes con tiempos anestésicos de más de 2 horas fue 16.49\% (16 de 97). Estos resultados nos orientan a pensar que el riesgo de presentar RRNM será mayor en pacientes que tengan tiempos anestésicos de una hora o menos, ya que regularmente se les aplica una dosis única de relajante para la intubación y el anestesiólogo asume que el efecto de éste ya ha pasado. Los pacientes con tiempo anestésicos mayores, por obvias razones, recibirán dosis subsecuentes del fármaco, lo que obliga al anestesiólogo a administrar con más cuidado las dosis adicionales.

\section{Conclusiones y trabajo futuro}

La combinación del algoritmo, con el operador de aceleración, el mecanismo de mejora y la función de evaluación basada en testores, fue capaz de resolver eficientemente el problema de la determinación del conjunto completo de los testores típicos de una base de datos; problema para el que los algoritmos exhaustivos conocidos tienen una complejidad exponencial [17].

La identificación de los 219 testores típicos inmersos en los datos, permitió realizar el cálculo del peso informacional de cada una de las variables, con la consecuente discriminación de aquellas cuyo peso informacional se encontraba por debajo del $29 \%$. Este punto de corte fue establecido por el personal médico especializado en anestesiología que formó parte del equipo de trabajo de la presente investigación.

Las variables que se encontraron por encima de este punto de corte fueron 10, lo que representa una reducción del $61.5 \%$ para considerarse como principales factores predisponentes en la relajación residual neuromuscular.

Como trabajo futuro, se incluirá una comparación de los resultados de este análisis con técnicas estadísticas clásicas, así como la integración de los mecanismos descritos en un Algoritmo de Estimación de la Distribución. Otro aspecto que se pretende agregar a la herramienta es el establecimiento automático del punto de corte. El equipo de trabajo también seguirá probando las herramientas con otros problemas médicos.

\section{Referencias}

1. Alba-Cabrera, E., Santana, R., Ochoa-Rodríguez, A., Lazo-Cortes. M.: Finding Typical Testors by Using an Evolutionary Strategy. In: Proceedings of V Iberoamerican Workshop on Pattern Recognition, Lisbon, Portugal, pp. 267-278 (2000)

2. Bermúdez, L.: Incidencia de la Relajación Residual Neuromuscular en la Unidad de Cuidados Intensivos. Tesis inédita de Especialidad Médica en Anestesiología, Universidad Autónoma de Aguascalientes, Ags, México (2015)

3. Cheguis, I. A., Yablonskii, S. V.: About testors for electrical outlines. Uspieji Matematicheskij Nauk, 4(66), pp. 182-184, Moscow (In Russian) (1955)

4. Dash, M., Liu, H.: Feature selection for classification. Intelligent Data Analysis, Vol. 1, pp. 131-156 (1997)

5. Dmitriev, A. N., Zhuravlev, Yu. I., Krendeleiev, F. P.: On the mathematical principles of patterns and phenomena classification. Diskretnyi Analiz, 7, pp. 3-15, Novosibirsk, Russia (In Russian) (1966) 
6. Dy, J. G. and Brodley, C. E.: Feature Selection for Unsupervised Learning. The Journal of Machine Learning Research. Vol 5. Publisher MIT Press. Cambridge, MA, USA. ISSN: 1533-7928. pp. 845-889 (2004)

7. Murphy, S.G.: Residual neuromuscular blockade and critical respiratory events in the postanesthesia care, Anesthesia and analgesia, vol. 32, pp. 107-130 (2008)

8. Goldberg D. E.: Genetic Algorithms in Search, Optimization, and Machine Learning, New York. Addison-Wesley (1989)

9. Holland J. H.: Adaptation in Natural and Artificial Systems: An Introductory Analysis with Applications to Biology, Control, and Artificial Intelligence, Ann Arbor. The University of Michigan Press. 1975. Second edition. Cambridge, MA: The MIT Press (1992)

10. Kohavi R. G. H. Jhon.: Wrappers for Feature Subset Selection. Artificial Intelligence, 97. pp 273-324. (1997)

11. Lazo-Cortés M., Ruiz-Shulcloper J.: Determining the feature relevance for non classically described objects and a new algorithm to compute typical fuzzy testors Pattern Recognition Letters, vol. 16, pp. 1259-1265 (1995)

12. Lazo-Cortes, M., Ruiz-Shulcloper, J., Alba-Cabrera, E.: An Overview of the Evolution of the Concept of Testor. Pattern Recognition, vol. 34, pp. 753-762 ( 2001)

13. Mierswa, I., Wurst, M.: Information Preserving Multi-Objective Feature Selection for Unsupervised Learning. Proc. of the Genetic and Evolutionary Computation Conference (GECCO 2006), pp. 1545-1552, New York, USA, ACM Press.(2006)

14. Miller E,L. RD: Anestesia de Anestesia, Elsevier, pp. 811-821 (2000)

15. Peng L., Jiaxian Z., Lanjuan L., Yanhong L., Xuefeng Z.:Data mining application in prosecution committee for unsupervised learning. Services Systems and Services Management, 2005. Proceedings of ICSSSM '05. 2005 International Conference on. Volume 2, 13-15, pp 1061 - 1064 Vol. 2. (2005)

16. Salomé B., Viana C.E., Cohelo V., Silva, A.: Bloqueo Neuromuscular Residual después del Uso del Rocuronio o Cisatracúrio, Revista Brasileña de Anestesiología, 55: 6: pp 612616, (2005)

17. Sanchez-Diaz, G., Lazo-Cortes, M.: CT-EXT: An Algorithm for Computing Typical Testor Set, Springer-CIARP07, pp 506-514, (2007). Proceedings. Lecture Notes in Computer Science 4756 Springer 2008, ISBN 978-3-540-76724-4, (2008)

18. Santiesteban, A. Y., Pons P. A.: LEX: Un Nuevo Algoritmo para el Cálculo de los Testores Típicos, Revista Ciencias Matemáticas, Vol.21, No.1, pp. 85-95. ISSN: 0256-5374. (2003)

19. Saeys, Y., Degroeve, S., Van de Peer, Y.: Digging into Acceptor Splice Site Prediction: An Iterative Feature Selection Approach. PKDD 386-397 (2004)

20. Shulcloper, J. R., Alba-Cortes, Lazo, C.: Introducción al reconocimiento de Patrones: Enfoque Lógico Combinatorio, Serie Verde, No. 51, CINVESTAV-IPN. México . pp. 188. (1995)

21. Shulcloper, J. R, Bravo M. A., Lazo-Cortes.: Algoritmos BT y TB para el cálculo de todos los test típicos. Revista Ciencias Matemáticas Vol. VI (2) pp.11-18. Cuba. (1985)

22. Torres M.D, Ponce E.E,Torres A, Luna F.J.: Selección de Subconjuntos de Características en Aprendizaje no Supervisado utilizando una Estrategia Genética en Combinación con Testores y Testores Típicos. Tercer Congreso Internacional de Computación Evolutiva (COMCEV 2007). ISBN: 9707280557. pp 57-63 ](2007)

23. Torres M. D. Metaheurísticas Hibridas en Selección de Subconjuntos de Características para Aprendizaje no Supervisado. (Tesis inédita de Doctorado). Universidad Autónoma de Aguascalientes, Ags, México (2010) 
María Dolores Torres Soto, Aurora Torres Soto, María de la Luz Torres Soto, et al.

YongSeog K., W. Nick Street, Menczer, F., Feature selection in unsupervised learning via evolutionary search. KDD: 365-369 (2000) 